\title{
Impact of Commitment on Performance Evaluation in the Rostral Cingulate Motor Area
}

\author{
Thomas Michelet, Bernard Bioulac, Dominique Guehl, Ludovic Escola, and Pierre Burbaud \\ Laboratoire de Physiologie et Physiopathologie de la Signalisation Cellulaire, Centre National de la Recherche Scientifique, Unité Mixte de Recherche 5543, \\ Université Victor Segalen Bordeaux2, 33076 Bordeaux, France
}

\begin{abstract}
Performance evaluation is a prerequisite for behavioral adaptation. Although the anterior cingulate cortex (ACC) is thought to play a central role in error detection, little is known about the electrophysiological activity of this structure during the performance-monitoring process. We directly addressed this issue by training monkeys to perform a Stroop-like task and then recorded neuronal activity in the rostral cingulate motor area (CMAr), a relatively unexplored region of the ACC known to be involved in motor processing. We found that most CMAr neurons responded during the evaluation period to both positive and negative feedback, but neuronal changes were more important after an error than after a successful trial. Interestingly, this performance-monitoring activity was not directly modulated by the degree of difficulty of the cognitive situation because changes in discharge frequency were similar whatever the level of attentional control imposed on the monkey. Firing activity during the evaluation period increased more, however, in erroneously completed than in incompleted trials and when the reward was delivered in an active rather than passive context, indicating that performance evaluation was conditioned by the degree of commitment of the animal to the task. It would thus seem that CMAr neurons could constitute a system for the evaluation of behavioral performance contingent on the subject's commitment to the task.
\end{abstract}

Key words: anterior cingulate cortex; performance monitoring; single-unit recording; monkey; error detection; cingulate motor area

\section{Introduction}

Among the different cognitive processes, performance evaluation is a mechanism related to some of the more fundamental components of behavior such as learning, adaptation, or selfregulation. A particularly good example of this monitoring function is the error-related negativity (ERN), a sharp negative deflection in event-related brain potential (ERP) that has been shown to occur in humans just after a person has committed an error, whether or not the error is indicated by negative feedback (Gehring et al., 1990; Falkenstein et al., 1995; Miltner et al., 1997). Dipole analysis of human brain ERP, electrophysiological data from primates, and, more recently, human neuroimaging studies all converge to situate the neural source of the ERN in the anterior cingulate cortex (ACC), an area already known to be implicated in reward assessment and thus involved in positive reinforcement circuitry (Bush et al., 2002; Shidara and Richmond, 2002; Hadland et al., 2003; Amiez et al., 2005, 2006). The ACC is also a pivotal region that receives numerous inputs from cognitive (Morecraft and Van Hoesen, 1993; Lu et al., 1994) and motivational (Morecraft and Van Hoesen, 1998) sources and projects in

\footnotetext{
Received 0ct. 30, 2006; revised May 28, 2007; accepted May 31, 2007.

This work was supported by the Ministère Délégué a la Recherche et aux Nouvelles Technologies as part of the AC "Neurosciences Intégratives et Computationnelles" program. T.M. is also supported by the Fondation pour la Recherche Médicale. We thank S. Dovero for histological preparation, M. Goillandeau for developing our software, and J. Simmers for his helpful comments on this manuscript.

Correspondence should be addressed to Thomas Michelet, Université de Montréal,Département de Physiologie, Pavillon Paul-G.-Desmarais, Université de Montréal, C.P. 6128, succursale Centre-ville, Montréal, Quebec, Canada H3C 3J7. E-mail: thomas.michelet@umontreal.ca.

D01:10.1523/JNEUROSCI.4718-06.2007

Copyright $\odot 2007$ Society for Neuroscience $\quad$ 0270-6474/07/277482-08\$15.00/0
}

turn to premotor and motor structures (Dum and Strick, 2002). It has consequently been proposed that the ACC may play an important role in regulating the impact of cognitive information on motor control in changing motivational states (Paus, 2001). This would explain, in part at least, why ACC dysfunction appears to play such an important role in certain neuropsychiatric conditions such as obsessive compulsive disorder. Although a number of functional imaging studies have been performed in humans, little is known of the actual activity of ACC neurons during performance evaluation. Reward- or error-linked modifications in neuronal activity have, however, been described, and it was found recently that ACC error-related activity was modulated by predicted reward (Amiez et al., 2005). What has not yet been investigated is the impact of cognitive information on the action-evaluation process and whether the type of error or the degree of commitment to the task modify this process.

To address this issue, we trained two monkeys to perform a Stroop-like visuomotor task that favors the execution of an incorrect response and allows assessment of cognitive factors. We focused on the rostral cingulate motor area (CMAr), a mediodorsal subregion of the ACC found in both human and nonhuman primates (Picard and Strick, 2001) that is thought to play a critical role in the generation of error-related neuronal activity (Gemba et al., 1986; Holroyd and Coles, 2002). We restricted our analysis to data obtained during the period of performance evaluation only.

\section{Materials and Methods}

Animals. Recordings were conducted in two female monkeys (Macaca mulatta), weighing 5 and $6 \mathrm{~kg}$, respectively. The animals were housed in 
A

C
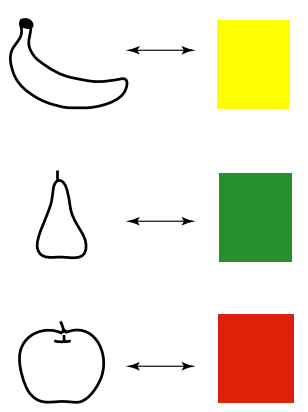

B

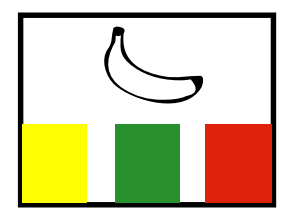

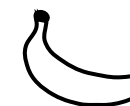
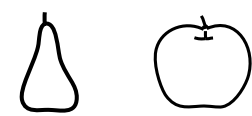

Level of difficulty - Intermediate (control situation)
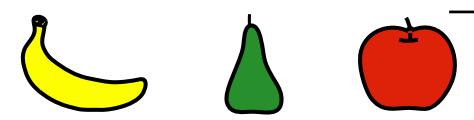

Level of difficulty - easy
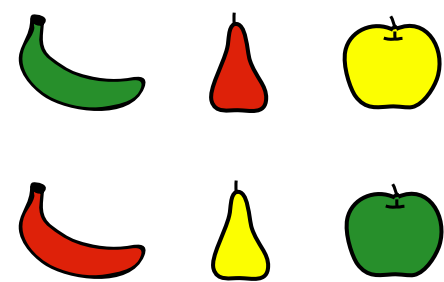

Level of difficulty - difficult

Figure 1. Composition of our Stroop-like task. $A$, Monkeys were taught to associate each fruit shape with its normal color. $B$, Shapes and colors were then assembled to form different cognitive situations, of three different levels of difficulty.

individual primate cages, and their care was supervised by veterinarians skilled in the health care and maintenance of nonhuman primates, in strict accordance with the European Community Council Directive for experimental procedures in animals.

Experimental paradigm. We designed an experimental paradigm based on the original Stroop test that engages different and quantifiable attentional demands. The Stroop-like task (SLT) we devised associated learning the shape of a fruit (banana, apple, or pear) and its color (yellow, red, and green, respectively; size, $5 \times 9 \mathrm{~cm}$ ) (Fig. $1 \mathrm{~A}$ ). When the three colors were presented on a video touch screen, monkeys had to touch the one that corresponded to the fruit shape simultaneously presented (Fig. $1 B$ ). By permuting color and shape, we were able to present three cognitive situations, corresponding to three levels of difficulty (Fig. 1C): (1) a basic control situation in which the shape is colorless and the task is of an intermediate level of difficulty, (2) a situation in which shape and color correspond (e.g., the banana is yellow) and the task is easy, and (3) a situation in which there is no congruence (i.e., interference) between color and shape (e.g., the banana is red) and the task is difficult.

During experimental recording sessions, monkeys were seated in a primate chair, their heads were immobilized, and their left hands were restrained, facing a video touch screen. Animals were trained to keep their right hands on the hand rest, which integrated a position sensor, until the shape and three colors appeared on the screen. Each trial comprised the same succession of events (Fig. 2A,B) (1) a $3 \mathrm{~s}$ rest period during which the screen remained white and the monkey was instructed to remain still, (2) a $500 \mathrm{~ms}$ warning period (presentation of a warning stimulus in the form of a black circle, diameter of $5 \mathrm{~cm}$ ), (3) after 500$1000 \mathrm{~ms}$, presentation of the task itself (SLT), i.e., the simultaneous presentation of one fruit shape and the three different colors, (4) the response period during which the monkey took its hand off the hand rest, thus releasing the position sensor, and was supposed to touch the correct color on the screen, and (5) the evaluation period that began with either delivery of the reward (a drop of orange juice) in the case of success or the appearance of a negative visual feedback in the form of a black screen after an incorrect response.

To exclude the possibility that the neuronal response to the negative feedback signal could be a simple visual response, we interspersed at random other trials in which we presented a colored screen (pink, blue, or brown) immediately after the initial rest period. In the same way, we checked that neuronal activity related to obtainment of the reward was not attributable to simple appetitive mechanisms by occasionally giving drops of orange juice immediately after the rest period. We also charted the activity of the biceps brachii and triceps brachii muscles and used a custom-made video apparatus to record oculomotor activity. The previous training of each monkey took $\sim 6$ months. This began with the learning of a simple motor task, which was subsequently used during recording sessions as a motor control test, in which monkeys had to touch a black target, displayed randomly on the left, in the middle, or on the right of a video touch screen. Once they had mastered this stage, we confronted them with the basic control situation, in which the three colors were presented with a colorless fruit shape. When a monkey's overall performance reached $>50 \%$ correct responses (chance, $33 \%$ ), we proposed the easy situation, in which the color of the fruit presented was its normal color. Once this stage was mastered according to the same criteria, we moved on to the difficult situation. The 12 different possible combinations (Fig. 1) were presented randomly within each recording session, with the color order of targets ( six possibilities, e.g., from left to right: red, green, and yellow) being changed for each recording session.

Surgery and electrophysiological recordings. A stainless steel recording chamber (diameter of $19 \mathrm{~mm}$ ) was implanted in the skull of each monkey under general anesthesia (ketamine, $10 \mathrm{mg} / \mathrm{kg}$; xylazine, $2 \mathrm{mg} / \mathrm{kg}$; diazepam, $0.5 \mathrm{mg} / \mathrm{kg}$; and atropine sulfate, $0.2 \mathrm{mg} / \mathrm{kg}$ ). Supplemental doses of ketamine were given when necessary to maintain a state of deep anesthesia. The central axis of the cylinder was stereotactically positioned at A26 and L0 in both monkeys. A head holder was embedded with dental cement around the chamber for immobilization during neuronal recording. Antibiotic (ampicillin, $100 \mathrm{mg} / \mathrm{kg}$ ) and analgesic (paracetamol, 30 $\mathrm{mg} / \mathrm{kg}$ ) treatments were given for 1 week after surgery. Extracellular single-unit activity was recorded using tungsten microelectrodes insulated with epoxy (impedance, $0.5-1.0 \mathrm{M} \Omega$ at $1 \mathrm{kHz}$ ). Neuronal activity was amplified $(10,000-20,000)$, filtered $(300-3000 \mathrm{~Hz})$, and displayed on an oscilloscope. A window discriminator was used to select spikes from background activity. These were then processed through an analogto-digital converter interface and stored on-line in a microcomputer. Analyses were performed on successive $10 \mathrm{~ms}$ data bins using customwritten software.

Behavioral statistical analysis. Three behavioral parameters were studied: error type (ET), error rate, and reaction time (RT) for the three cognitive situations.

For error type, we listed the five types of incorrect response that could elicit the appearance of the visual negative feedback signal (Fig. 2 B): (1) a no movement error (ET1), in which the monkey did not move at all during the trial; (2) an early response error (ET2), in which the trial was interrupted because the monkey moved too early (i.e., before the SLT appeared on the screen); (3) an invalid response error (ET3), in which the monkey responded but either touched the screen with insufficient force to validate the trial (minimum touch activation force necessary, 55-85 g) or stopped before actually touching the screen; (4) an incorrect response error (ET4), in which the monkey touched the wrong color (e.g., the red target in response to the presentation of the banana shape); and (5) a too late response, in which the monkey lifted its hand after the $4 \mathrm{~s}$ of the response period. There were too few trials of this last error type to allow statistical analysis.

Error rate was calculated for all trials in which the monkey completed the response movement, i.e., touched a target on the screen with sufficient force to validate the trial. We therefore excluded type 1,2 , and 3 errors from this calculation. Because type 5 errors had not been retained for analysis, this left only type 4 errors. The number of erroneous trials was divided by the total number of completely executed trials for each of the three cognitive situations.

RT was defined as the interval between the moment when the SLT appeared on the screen and the moment when the monkey released the position sensor by lifting its hand off the hand rest (Fig. $2 \mathrm{~B}$ ). Again, we could only study this parameter for completed movements and therefore restricted our analysis to successful trials and type 4 errors (the monkey touched the wrong target). We then performed a two-way ANOVA for the three cognitive situations, taking as the dependent variable the mean 
RT calculated for the total number of sessions taken into account. For post hoc analysis, we used a Fisher's test with Bonferroni's/Dunn's correction.

Electrophysiological statistical analysis. We only analyzed data from neurons that were activated during a sufficient number of trials to make it possible to constitute a representative statistical sample. Perievent raster displays and histograms of neuronal activity were aligned to the beginning of the evaluation period, i.e., either reward delivery or appearance of the negative feedback signal. We then analyzed neuronal activity for a $500 \mathrm{~ms}$ epoch. This time window was calculated to encompass the classically described epoch during which evaluation-related neuronal activity peaks after a correct or incorrect response. Event-related modifications of activity were detected by comparing (paired $t$ test) neuronal activity related to a specific event with activity during the initial $3 \mathrm{~s}$ rest period. A change in neuronal discharge frequency was considered significant if the $p$ value was $<0.01$. To assess precisely the influence of the cognitive situation on performance-monitoring activity, we restricted our analysis to those trials during which animals made strictly comparable movements, effectively touching a target on the screen. We therefore compared successfully completed trials with type 4 errors (the monkey touched the wrong target) and excluded erroneous trials of types 1,2 , and 3. Because it was difficult to quantify inhibitory unitary responses, only neurons responding by an activation were analyzed. An ANOVA was then performed for the three cognitive situations. The dependent variable was the mean firing frequency calculated during the $500 \mathrm{~ms}$ epoch for all recorded neurons. We used a Fisher's test with Bonferroni's/Dunn's correction for post hoc analysis.

Histology. Once experimentation was completed, the boundaries of the region recorded were delimited by electrolytic lesion using a direct anodal current $(20 \mu \mathrm{A}, 20 \mathrm{~s})$ applied through a microelectrode. One week later, each animal was deeply anesthetized (Nembutal, $100 \mathrm{mg} / \mathrm{kg}$ ) and perfused through the ascending aorta with $500 \mathrm{ml}$ of $0.9 \%$ saline, followed by 2 liters of $4 \%$ paraformaldehyde in phosphate buffer, $\mathrm{pH} 7.4$, as fixative. The outline of the recording chamber was then traced on the surface of the brain, and the brain was removed from the skull and sliced into $25 \mathrm{~mm}$ frontal sections that were postfixed for $12 \mathrm{~h}$ at $4^{\circ} \mathrm{C}$ in $20 \%$ sucrose in Tris-buffered saline, $\mathrm{pH}$ 7.4, frozen in isopentane cooled on dry ice, and cut into $25 \mu \mathrm{m}$ frontal sections with a cryostat. All sections were counterstained with cresyl violet and then rinsed in PBS, dehydrated in gradually increasing concentrations of ethanol, cleared in xylene, and coverslipped in Neoantelan (Polylabo, Strasbourg, France). Recording sites were located using the coordinates of each recorded cell with respect to marker lesions and, when possible, to electrode tracks (Fig. 3). CMAr boundaries were determined using information obtained by intracortical microstimulation performed after each neuronal recording and sulcal landmarks and compared with previously determined magnetic resonance imaging (MRI) localization.

\section{Results}

\section{Behavioral results}

Error rate and mean reaction time for the three different situations, easy, intermediate level of difficulty (control situation), and difficult, are given in Figure 4 .

A $\chi^{2}(p<0.01)$ test revealed a positive correlation between error rate and the level of cognitive difficulty. These results con-

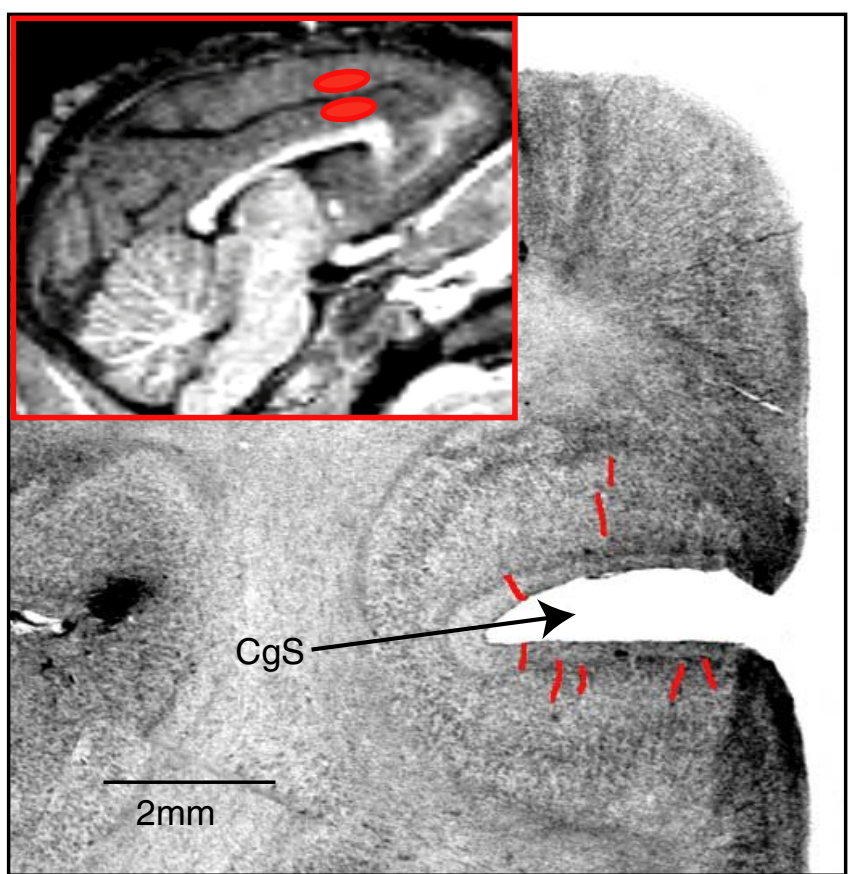

Figure 3. Location of single neuron recording sites. Regions of the upper and lower banks of the cingulate sulcus (CgS) sampled. Individual electrode paths are indicated by red lines in a coronal histological section taken at level A28. Inset is a mediosagittal MRI scan showing recorded regions (in red). 


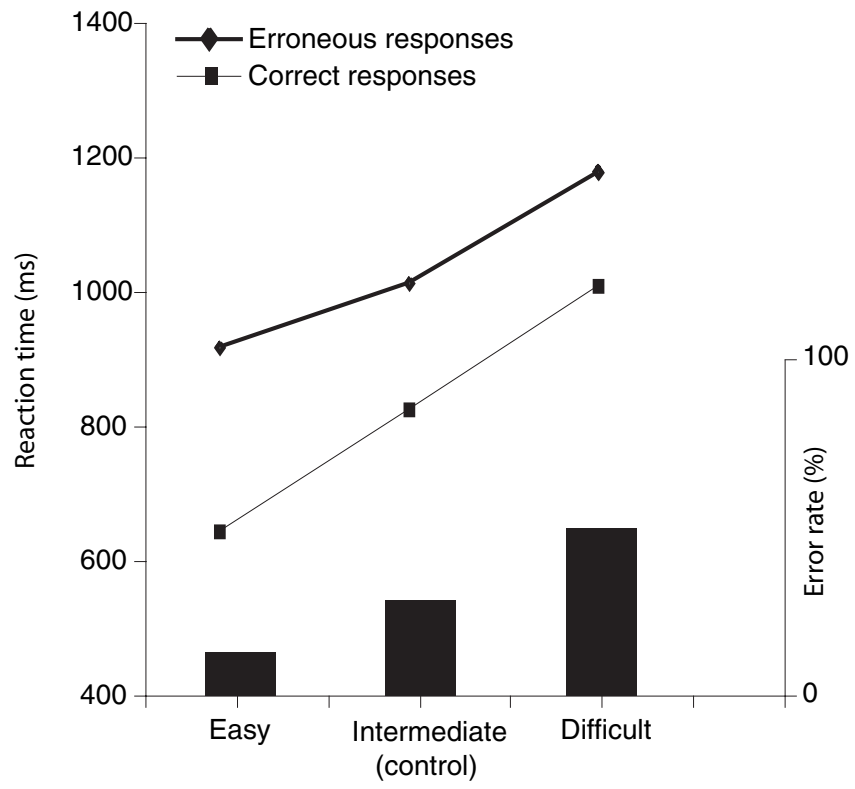

Figure 4. Mean behavioral performance for trials (correct responses or errors of type 4) during which there was significant neuronal activity during the evaluation period ( $n=92$ ). Mean reaction time increased with the level of difficulty, confirming that behavioral cost is a function of the level of cognitive control. Error rate (bars) increased significantly as the level of cognitive difficulty augmented.

firmed that the task chosen for this experiment allowed effective manipulation of behavioral parameters.

A two-way ANOVA on the three situations showed that the level of cognitive difficulty had a clear influence on $\mathrm{RT}\left(F_{(1,92)}=\right.$ $6.82 ; p<0.005)(p<0.0001)$. This was confirmed by post hoc comparison $(p<0.0001)$, which showed that $\mathrm{RT}$, expressed in milliseconds, increased significantly from the easy to the intermediate (control) to the difficult situation $(642,824.9$, and 1009.8 , respectively). The same analysis performed for erroneous trials only revealed a similar tendency $\left(F_{(1,92)}=3.3 ; p<0.05\right)$ for the three situations $(918.1,1014$, and 1179.7). A systematic comparison of RT in successful and in erroneous trials indicated a significant increase in RT ( $t$ test, $p<0.001$ ) for erroneous trials in each of the three situations.

\section{Unit recordings}

A total of 92 of the 183 ACC neurons recorded (50.2\%) exhibited significant activity during the evaluation period. Among these, we distinguished three groups. The activity of $11 \%$ of the 92 (group 1, $n=10$ ) was modified (eight activations and two inhibitions) by positive but not by negative feedback (Fig. 5A). Within this group, $70 \%(n=7)$ did not respond at all to positive feedback (reward delivery) outside the context of the SLT. A total of $18.4 \%$ of the 92 neurons (group 2, $n=17$ ), in contrast to the first group, increased their firing rate after negative but not after positive feedback (Fig. 5B). The third group (70.6\% of the 92 ) was the most frequent $(n=65)$, with a complex pattern of activation and/or inhibition during the evaluation period, as can be seen in Table 1 . Most of these neurons $(75 \%, n=49)$ showed a strikingly phasic activation to negative feedback, as well as displayed a smaller but significant transient increase in activity after positive feedback (Fig. 6). Reward and visual control tests (see Materials and Methods) for any given neuron confirmed that simple visual or appetitive mechanisms could not account for these responses. When the mean peak spike frequencies of the 65 neurons of this third group were pooled, mean \pm SEM response magnitude
A

B
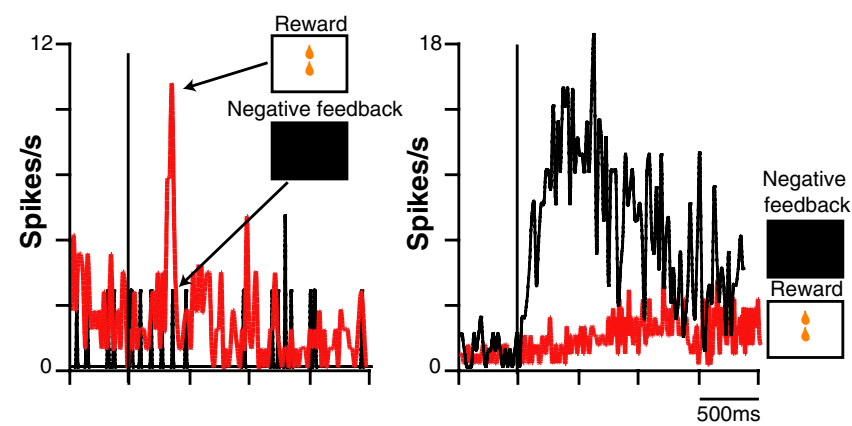

Figure 5. Typical examples of the mean activation of an individual CMAr neuron responding only to positive or only to negative feedback. $A$. Activation of a group 1 neuron that responded only to positive feedback (in red; $n=72$ ) and not to negative (in black; $n=12$ ). $\boldsymbol{B}$, Activation of a group 2 neuron that responded only to negative (in black; $n=65$ ) and not to positive (in red; $n=111)$ feedback.

Table 1. Distribution of neurons (group $3 ; n=65$ ) that responded to both positive and negative feedback by activation, inhibition, or both

\begin{tabular}{lccccc}
\hline $\begin{array}{l}\text { Positive feedback (success)/negative } \\
\text { feedback (error) }\end{array}$ & Total units & Success $>$ error & Error > success & NS \\
\hline Activation/activation & 49 & 8 & 33 & 8 \\
Activation/inhibition & 4 & 4 & 0 & 0 \\
Inhibition/activation & 8 & 0 & 8 & 0 \\
Inhibition/inhibition & 4 & & & \\
Total & 65 & 12 & 41 & \\
\hline
\end{tabular}

Trials in which the positive feedback (reward) was given out of the task context were excluded. NS, Not significant

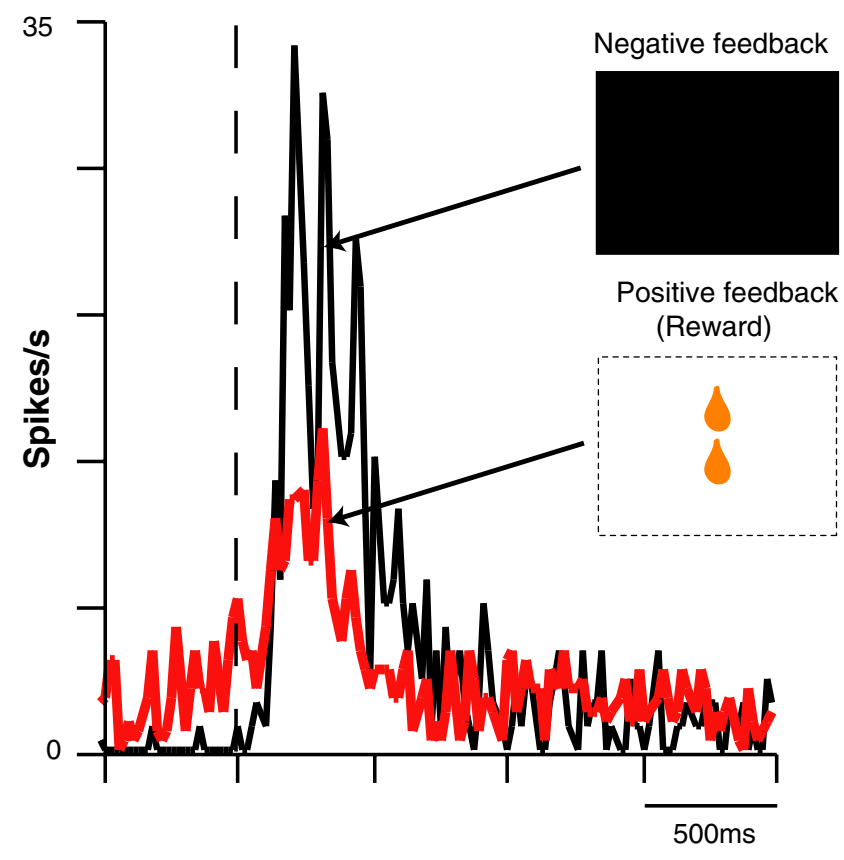

Figure 6. Neurons that responded to both positive and negative feedback (group 3) presented a more important activation after negative than after positive feedback. Example of an individual group 3 neuron. Firing rate increased more after negative (in black; $n=43$ ) than after positive (in red; $n=104$ ) feedback.

(spikes per second) was $47 \%$ higher after negative feedback $(12.6 \pm 2.6)$ than after reward delivery $(8.5 \pm 1.4)$, indicating a clear bias toward negative feedback signaling.

A one-way ANOVA on the 61 neurons activated by positive 
feedback (groups 1 and 3) for the three cognitive situations showed that only three neurons modified their activity according to the difficulty of the situation. The vast majority ( $90 \%, n=58)$ showed no significant modulation from one cognitive situation to another, as demonstrated by their virtually identical activity patterns (Fig. 7). For the 74 neurons that were activated by negative feedback (groups 2 and 3), the same analysis showed that only two neurons modified their activity according to the cognitive situation ( $p<0.01$; see Materials and Methods). Again, the vast majority $(93 \%, n=69)$ showed no significant modulation of their firing rate between the three situations. These results clearly indicate that cognitive information did not modify the discharge properties of the large majority of CMAr neurons involved in performance monitoring.

We then compared the mean firing rate of the 49 neurons that were activated by both positive and negative feedback after positive (completed correct response) and after negative (completed but incorrect response) feedback. A total of $83 \%(n=41)$ showed significant differences between evaluation of success and evaluation of error. A majority, 67\% $(n=33)$, modified their firing rate more after negative than after positive feedback. A reward given out of the task context induced a significantly $(p<0.001)$ smaller activation than a reward given after a successfully completed task (Fig. $8 \mathrm{~A}$, results for an individual neuron; $C$, white bars, pooled data for all neurons that responded to positive feedback).

To conclude, we looked at the discharge rate of evaluationrelated neurons as a function of the four different error types defined above (see Materials and Methods, Behavioral statistics analysis) (no movement ET1, premature response ET2, invalid response ET3, and incorrect response ET4). A one-way ANOVA showed a high level of significance $\left(F_{(3,74)}=6.22 ; p<0.0001\right)$, and post hoc analysis showed that mean discharge rate (spikes per second) increased gradually from ET1 to ET4 (respectively, 8.4, $9.08,10.39$, and 12.55) with significant differences between ET1/ ET2 and ET4. The slight difference observed $(p=0.05)$ between ET3 and ET4 was not statistically significant (Fig. 8C, black bars). Figure $8 B$ presents the activation of an individual neuron during the evaluation period in response to the negative feedback consequent to the four different error types.

\section{Discussion}

We devised for this study an original Stroop-like task that allowed us to investigate the role played by the CMAr in performance evaluation. The task required precise behavioral control, as was demonstrated by the high error rate and the fact that both error rate and reaction time varied considerably from one cognitive situation to another. This is additionally supported by our finding that RTs for errors were generally longer than RTs for correct responses. Indeed, this result can be explained by making the distinction between execution failure (such as slips or lapses) and planning failures (mistakes) (Reason, 1990a,b). Although the
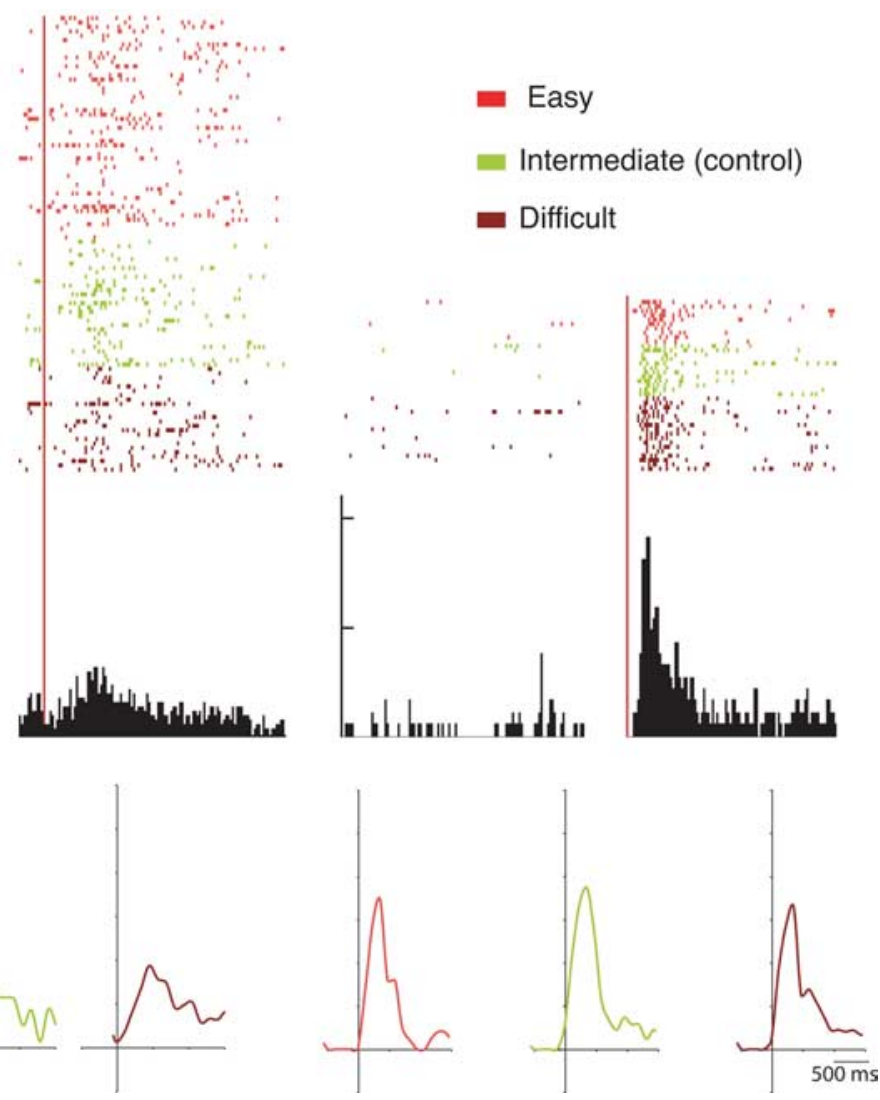

Figure 7. Activity of a typical CMAr neuron during the evaluation period in the three cognitive situations. Raster display showing firing frequency after positive (top left) and after negative (top right) feedback. Response profiles (after positive feedback, bottom left; after negative feedback, bottom right) were similar, whatever the cognitive situation.

former results from skilled-based components (such as mistiming) and are usually found in speeded RT task with a drastic RT deadline, the later are rather attributable to cognitive or rulebased difficulty. Because there was no drastic time constraint in the SLT, our data suggest that, when faced with cognitive difficulty, monkeys developed a conservative strategy to respond as accurately as possible.

Our finding that CMAr neuronal activity increased during the evaluation period is consistent with previous human and primate studies that have shown that ACC neurons are activated during reward ingestion or on the appearance of a positive feedback signal (Akkal et al., 2002; Isomura et al., 2003; Matsumoto et al., 2003; Ullsperger and von Cramon, 2003). We also found that more CMAr neurons were activated, and discharge rate increased, when feedback was negative. This indicates an implication in error detection that was first suggested 20 years ago, in primate studies that showed that ACC neurons were activated when animals committed an error or did not receive a reward to which they felt entitled (Niki and Watanabe, 1979; Gemba et al., 1986). These data have recently been endorsed by electrophysiological and neuroimaging data obtained in humans, which have provided new evidence that ACC activity is modulated by negative feedback (Ullsperger and von Cramon, 2003; Holroyd et al., 2004). Because single-cell recording remains the most direct technique for anatomo-functional localization, the data we present showing an activation of CMAr neurons during the evaluation period would confirm that this structure plays an important role in performance evaluation (Ito et al., 2003; Amiez et al., 2005, 2006). 
A

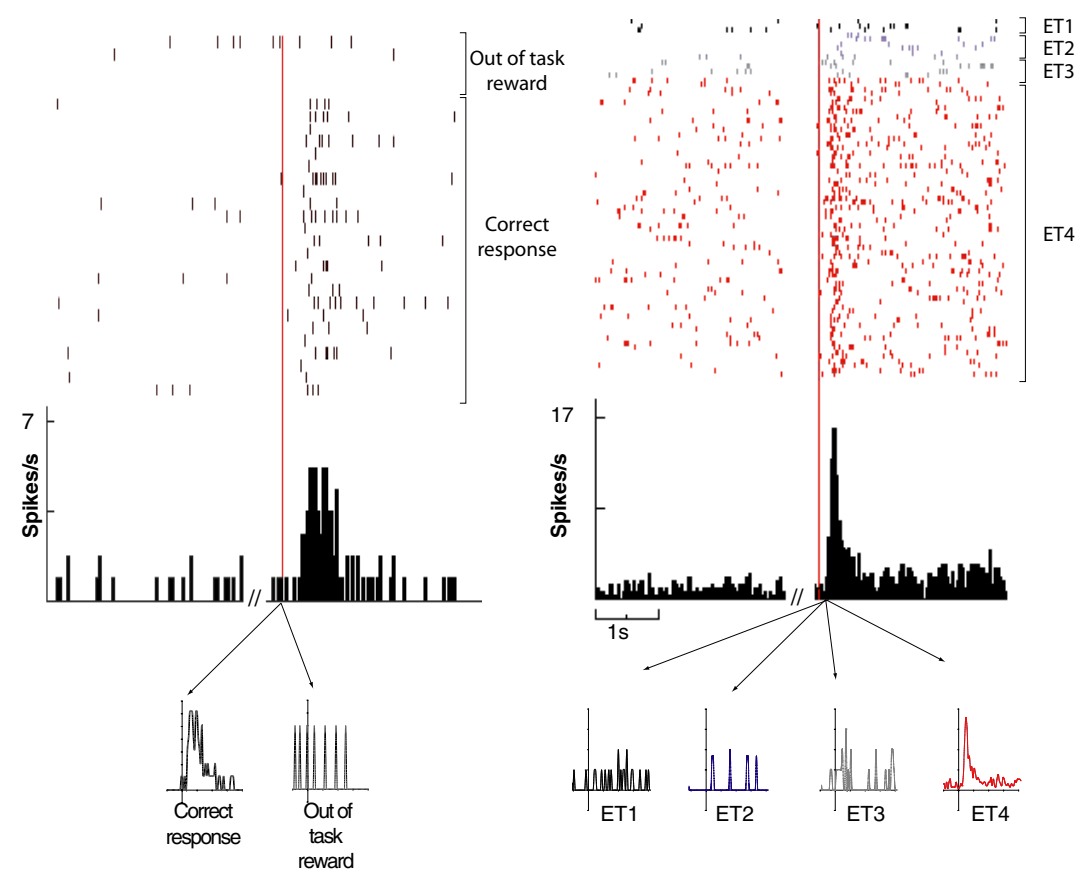

C

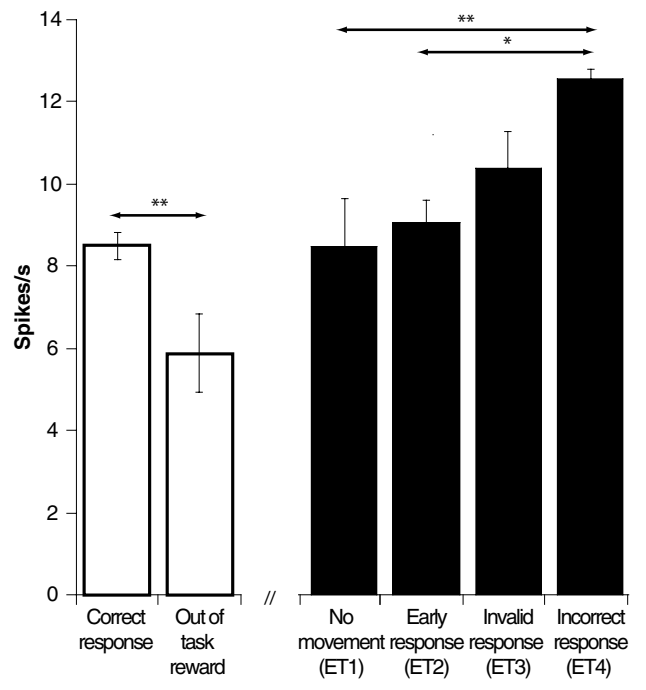

Figure 8. CMAr neuronal activation during the evaluation period was contingent on animal's commitment to the task. $\boldsymbol{A}$, Top represents raster display and frequency histogram comparing firing activity of an individual neuron during the initial $3 \mathrm{~s}$ rest period and during the evaluation period after positive feedback pooled for both correct response and out of the task reward. Bottom represents in two separate curves the mean activity for correct response and out of the task reward. $\boldsymbol{B}$, Top represents raster display and histogram comparing firing activity of an individual neuron during the initial $3 \mathrm{~s}$ rest period and during the evaluation period after the negative feedback engendered by the pooled four types of error analyzed. Bottom represents in four separate curves the mean activity of each of the four error types. C, White bars, Mean firing activity during the evaluation period of all neurons that responded to positive feedback. Activation was more important after positive feedback (reward delivery) consecutive to a correct execution of the SLT than after a reward delivered out of the task context. Black bars, Mean firing activity during the evaluation period of all neurons that responded to negative feedback, according to error type. The degree of activation during the evaluation period after negative feedback varied according to the type of error. The greater the degree of commitment of the animal to the task, the more important the activation.

This role confirmed, we were interested to see what is the influence of the cognitive situation on this activity. Our results showed a positive correlation between behavioral cost, as measured by reaction time and error rate, and cognitive demand. We then compared the activity of CMAr neurons during the evaluation period immediately after the successful or erroneous execution of the SLT in the three different cognitive situations. There was surprisingly little variation in the modification of firing rate from one situation to another. This would suggest that cognitive demand does not exercise a significantly specific influence on evaluation-related activity. These results are also in agreement with previous studies that emphasize the absence of modulation of CMAr outcome activity during response competition tasks (Ito et al., 2003; Nakamura et al., 2005). Because error rates were significantly different from one cognitive situation to another, our results rule out, however, a direct influence of uncertainty. If there had been a direct influence, the differences in the probability of success between the three cognitive conditions should have induced related, albeit distinct, neuronal activity patterns. It has indeed been proposed by Holroyd and Coles (2002) that a complementarity exists between a medial frontal system involved in performance-monitoring and dopaminergic (DA) neurons involved in coding the discrepancies between actual and expected event outcomes (reinforcement learning theory). This interpretation is based on the finding that mesencephalic DA neurons code error in the prediction of reward: the phasic increases or decreases in DA neurons indicates that ongoing events are better or worse, respectively, than expected (error prediction theory)
(Schultz, 1998; Schultz and Dickinson, 2000). According to Holroyd and Coles (2002), the ERN reflects the arrival of this DA error signal on the ACC with an inverse relationship: these authors indeed assumed that the impact of the DA signals on the apical dendrites of neurons in the ACC modulates the amplitude of the ERN, such that phasic decreases in dopamine activity (indicating that ongoing events are worse than expected) are associated with large ERNs. In this view, it is then predicted that larger ERNs should be elicited by unexpected unfavorable outcomes than by expected unfavorable outcomes. According to this, recent functional imaging results showed that error-related activity is greater for low-error conditions than for the high-error conditions (Brown and Braver, 2005). We then obviously expected that, in the present experiment, there should be a larger neuronal amplitude response on easy trials (i.e., the low-error condition). However, because the dramatic increase in firing rate we found after the negative feedback was not influenced by the task difficulty, our results seem to be in part inconsistent with these previous theoretical approaches and findings in their present form (Holroyd and Coles, 2002; Brown and Braver, 2005). However, in the Brown and Braver task, parameters were dynamically controlled with the use of a staircase algorithm as to produce error rates that were constant for each experimental condition. Because it was not the case in our experiment, this could have made it more difficult for the monkeys to maintain different reward expectations on trials of different difficulty levels (Holroyd and Coles, 2002) or to learn whether greater error likelihood was associated with difficult or easy trials (Brown and Braver, 2005). 
Another explanation could be that the ERN is not just reflecting the function of a single ACC region (such as the CMAr) but may in fact reflect the broader activity of a performancemonitoring network implemented in the whole medial frontal cortex (MFC) and in the limbic system (Luu et al., 2003). This explanation is supported by the finding that the rostral part of the supplementary motor area is also involved in such a process (Brown and Braver, 2005). It has already been proposed that controversies about the role of the MFC in performance monitoring may in part have arisen because different researchers are discussing separate parts of the MFC (Rushworth et al., 2004). An indirect argument for the functional parcellation of the ERN is that it correlates with many aspects of human error processing (Gehring et al., 1993; Falkenstein et al., 2000) and varies in amplitude across experimental conditions although its latency seems to be very consistent (Falkenstein et al., 2000). This suggests that different subprocesses are recorded at the same time and that the whole EEG signal encompasses but consequently minimizes the impact of each of its components.

The view that the ERN is elicited by the impact of a reward prediction error signal carried by the mesencephalic dopamine system on ACC could be also revised in this context: it is indeed possible that the functional parcellation of the ERN and its modulation are underlined by a relative balance in neuromodulation between more than one neuromodulator. Interestingly, recent physiological evidence indicates that not only dopamine but also noradrenaline affect neuronal activity during performance monitoring (Riba et al., 2005). Because the pattern of these neurotransmitters afferent connections appear to be specific (Porrino and Goldman-Rakic, 1982), it could account for the heterogeneity in ERP and function MRI experiments found in different subregions of the MFC. Although the obvious question remaining is then how components of this ERN signal are coordinated, our results imply that outcome predictability does not affect the evaluative activity of CMAr neurons.

These findings would appear to be incompatible with previous postulates that ACC evaluative activity is directly modulated by cognitive information and by outcome predictability. In our study, CMAr neuronal activity was, certainly, modulated during the evaluation period, but this modulation appeared to depend on the degree of commitment to performance of the task. When the reward was obtained passively (i.e., out of the task context), we found a dramatic decrease in firing rate compared with the firing rate observed on obtainment of the reward after a correct response. The fact that CMAr neuronal activation was greater when a reward was given in the task context than out of context would in itself indicate that a large proportion of these neurons are reinforcement rather than reward related because, in reinforcement-related activity, the response to the reward is linked to the execution of a particular task, whereas in rewardrelated activity the neuronal reaction is the same, whatever the circumstances of reward delivery (Watanabe, 2002). In the same way, the "volition-to-action" transformation mechanism such as that suggested by Gehring et al. (1993) may shed some light on the differential activation of CMAr neurons during the evaluation period after the four types of error. These authors already proposed a few years ago that the integrative process manifested by the so-called ERN is influenced by the importance the subject attaches to the error he has perceived. More recently, other studies have pointed out that ACC activation at very early stages of information processing reflects the amount of effort or volition that a subject is willing or able to engage in a task (Winterer et al., 2002). This has also been indicated by experiments in which the ability to perform difficult tasks was impaired by lesioning the medial frontal cortex, including the ACC (Walton et al., 2002; Kennerley et al., 2006). Interestingly, in our experiments, the type of error after which the discharge rate varied least was the type 1 error, during which the monkey failed to initiate any movement. In an operant conditioning paradigm, the animal could hardly expect a positive outcome without executing the task. Because the context was strictly the same as in previous and subsequent successful trials, the virtual absence of a variation in discharge rate strongly suggests that the monkey was not engaged in the task. A change in firing activity would thus be less likely to be related to the failure to obtain a possible reward than to a conditional response to negative feedback. In type 2 erroneous trials (the monkey moved before the task was presented), it is likewise probable that the premature motor response reflected impulsivity or eagerness to act, which could explain the relatively small modification in firing activity observed during the evaluation period. In contrast, in the type 4 error, the execution motor program was appropriately activated to touch one of the three possible targets, even if a wrong target was finally selected. CMAr activation was more important during negative feedback after this type of error, indicating a higher level of commitment. We also observed a very slight variation in firing rate modulation between type 3 errors, when the monkey exerted insufficient pressure on the screen, and type 4 errors, when the monkey completed the movement but touched the wrong target. This could possibly be explained by the fact that, in type 3 errors, there may be a decrease in sensory feedback. The monkey either did not press hard enough or had a last-minute hesitation as to its choice and restrained its movement just as it was about to touch the target.

These observations lead us to conclude that the graded neuronal activation observed in the CMAr during performance evaluation is contingent on the execution of the motor response. In both successful and type 4 erroneous trials, the two cases in which the response movement was completely accomplished, the response was encoded in a standard manner. This was the case, whatever the cognitive context. These results confirm the precise role for CMAr neurons in linking voluntary actions and their outcomes (Shima and Tanji, 1998). The relative independence of CMAr neuronal activity to positive or negative feedback in the absence of overt movements should guarantee that these feedbacks have any relevance for performance of the task. It is possible that positive feedback after a success may help to reinforce the motor response through stabilization of the neuronal circuit implicated in the response. However, the fact that changes in neuronal discharge frequency were greater after an erroneous than after a successful response indicates that the CMAr is more solicited when the subject has to adapt its behavior. Such changing activity patterns may in turn be determinant for cognitive and motor learning by trial and error. Because the CMAr sends direct projections to the primary motor cortex and the spinal cord, as well as to the supplementary motor area, it is likely that errorrelated activity exerts a direct influence on motor programming and execution.

\section{References}

Akkal D, Bioulac B, Audin J, Burbaud P (2002) Comparison of neuronal activity in the rostral supplementary and cingulate motor areas during a task with cognitive and motor demands. Eur J Neurosci 15:887-904.

Amiez C, Joseph JP, Procyk E (2005) Anterior cingulate error-related activity is modulated by predicted reward. Eur J Neurosci 21:3447-3452.

Amiez C, Joseph JP, Procyk E (2006) Reward encoding in the monkey anterior cingulate cortex. Cereb Cortex 16:1040-1055. 
Brown JW, Braver TS (2005) Learned predictions of error likelihood in the anterior cingulate cortex. Science 307:1118-1121.

Bush G, Vogt BA, Holmes J, Dale AM, Greve D, Jenike MA, Rosen BR (2002) Dorsal anterior cingulate cortex: a role in reward-based decision making. Proc Natl Acad Sci USA 99:523-528.

Dum RP, Strick PL (2002) Motor areas in the frontal lobe of the primate. Physiol Behav 77:677-682.

Falkenstein M, Hohnsbein J, Hoormann J (1995) Event-related potential correlates of errors in reaction tasks. Electroencephalogr Clin Neurophysiol Suppl 44:287-296.

Falkenstein M, Hoormann J, Christ S, Hohnsbein J (2000) ERP components on reaction errors and their functional significance: a tutorial. Biol Psychol 51:87-107.

Gehring WJ, Coles MG, Meyer DE, Donchin E (1990) The error-related negativity: an event related potential accompanying errors. Psychophysiology 27:S34.

Gehring WJ, Goss B, Coles MG, Meyer DE, Donchin E (1993) A neural system for error detection and compensation. Psychol Sci 4:385-390.

Gemba H, Sasaki K, Brooks VB (1986) "Error" potentials in limbic cortex (anterior cingulate area 24) of monkeys during motor learning. Neurosci Lett 70:223-227.

Hadland KA, Rushworth MF, Gaffan D, Passingham RE (2003) The anterior cingulate and reward-guided selection of actions. J Neurophysiol 89:1161-1164.

Holroyd CB, Coles MG (2002) The neural basis of human error processing: reinforcement learning, dopamine, and the error-related negativity. Psychol Rev 109:679-709.

Holroyd CB, Nieuwenhuis S, Yeung N, Nystrom L, Mars RB, Coles MG, Cohen JD (2004) Dorsal anterior cingulate cortex shows fMRI response to internal and external error signals. Nat Neurosci 7:497-498.

Isomura Y, Ito Y, Akazawa T, Nambu A, Takada M (2003) Neural coding of "attention for action" and "response selection" in primate anterior cingulate cortex. J Neurosci 23:8002-8012.

Ito S, Stuphorn V, Brown JW, Schall JD (2003) Performance monitoring by the anterior cingulate cortex during saccade countermanding. Science 302:120-122.

Kennerley SW, Walton ME, Behrens TE, Buckley MJ, Rushworth MF (2006) Optimal decision making and the anterior cingulate cortex. Nat Neurosci 9:940-947.

Lu MT, Preston JB, Strick PL (1994) Interconnections between the prefrontal cortex and the premotor areas in the frontal lobe. J Comp Neurol 341:375-392.

Luu P, Tucker DM, Derryberry D, Reed M, Poulsen C (2003) Electrophysiological responses to errors and feedback in the process of action regulation. Psychol Sci 14:47-53.

Matsumoto K, Suzuki W, Tanaka K (2003) Neuronal correlates of goalbased motor selection in the prefrontal cortex. Science 301:229-232.

Miltner W, Braun C, Coles MG (1997) Event-related brain potentials fol- lowing incorrect feedback in a time-estimation task: evidence for a "generic" neural system for error detection. J Cogn Neurosci 9:788-798.

Morecraft RJ, Van Hoesen GW (1993) Frontal granular cortex input to the cingulate (M3), supplementary (M2) and primary (M1) motor cortices in the rhesus monkey. J Comp Neurol 337:669-689.

Morecraft RJ, Van Hoesen GW (1998) Convergence of limbic input to the cingulate motor cortex in the rhesus monkey. Brain Res Bull 45:209-232.

Nakamura K, Roesch MR, Olson CR (2005) Neuronal activity in macaque SEF and ACC during performance of tasks involving conflict. J Neurophysiol 93:884-908.

Niki H, Watanabe M (1979) Prefrontal and cingulate unit activity during timing behavior in the monkey. Brain Res 171:213-224.

Paus T (2001) Primate anterior cingulate cortex: where motor control, drive and cognition interface. Nat Rev Neurosci 2:417-424.

Picard N, Strick PL (2001) Imaging the premotor areas. Curr Opin Neurobiol 11:663-672.

Porrino LJ, Goldman-Rakic PS (1982) Brainstem innervation of prefrontal and anterior cingulate cortex in the rhesus monkey revealed by retrograde transport of HRP. J Comp Neurol 205:63-76.

Reason JT (1990a) The detection of errors. In: Human error, pp 148-172. Cambridge, UK: Cambridge UP.

Reason JT (1990b) Performance levels and error types. In: Human error, pp 53-96. Cambridge, UK: Cambridge UP.

Riba J, Rodriguez-Fornells A, Morte A, Munte TF, Barbanoj MJ (2005) Noradrenergic stimulation enhances human action monitoring. J Neurosci 25:4370-4374.

Rushworth MF, Walton ME, Kennerley SW, Bannerman DM (2004) Action sets and decisions in the medial frontal cortex. Trends Cogn Sci $8: 410-417$.

Schultz W (1998) Predictive reward signal of dopamine neurons. J Neurophysiol 80:1-27.

Schultz W, Dickinson A (2000) Neuronal coding of prediction errors. Annu Rev Neurosci 23:473-500.

Shidara M, Richmond BJ (2002) Anterior cingulate: single neuronal signals related to degree of reward expectancy. Science 296:1709-1711.

Shima K, Tanji J (1998) Role for cingulate motor area cells in voluntary movement selection based on reward. Science 282:1335-1338.

Ullsperger M, von Cramon DY (2003) Error monitoring using external feedback: specific roles of the habenular complex, the reward system, and the cingulate motor area revealed by functional magnetic resonance imaging. J Neurosci 23:4308-4314.

Walton ME, Bannerman DM, Rushworth MF (2002) The role of rat medial frontal cortex in effort-based decision making. J Neurosci 22:10996-11003.

Watanabe M (2002) Integration across multiple cognitive and motivational domains in monkey prefrontal cortex. In: Principles of frontal lobe function, pp 326-337. Oxford: Oxford UP.

Winterer G, Adams CM, Jones DW, Knutson B (2002) Volition to action: an event-related fMRI study. NeuroImage 17:851-858. 